\section{IN THE NEWS}

\section{Plane thinking}

A clump of rat neurons in a

Petri dish might not have the glamorous image that we

usually associate with airline pilots, but thanks to research at the University of Florida, it might soon be able to do their job just as effectively.

Biomedical engineer Thomas DeMarse has "created a living 'brain' of cultured rat cells that now controls an F-22 fighter jet flight simulator" (Discovery Channel, USA, 22 October).

DeMarse seeded a grid of 60 electrodes with 25,000 rat neurons, which were allowed to grow and form a neural network. This network was connected to a flight simulator, and the electrodes were used both to record the activity of individual neurons and to provide 'feedback' on their performance in the form of electrical stimuli.

According to DeMarse, "over time, these stimulations modify the network's response such that the neurons slowly (over the course of 15 minutes) learn to control the aircraft" (Discovery Channe).

Although it sounds like the stuff of science fiction, it is believed that this technology will one day have real practical applications. Mandayam Srinivasan from the Massachusetts Institute of Technology said, "there are certainly things that biological systems can accomplish that we haven't been able to do with electronics". For example, "animals have no problem recognising different textures or telling the difference between two different pieces of furniture, whereas computers find this very difficult" (New Scientist, UK, 25 October).

DeMarse admits, "we're just starting out. But using this model will help us understand the crucial bit of information between inputs and the stuff that comes out. And you can imagine the more you learn about that, the more you can harness the computation of these neurons into a wide range of applications" (Discovery Channel).

Heather Wood

\title{
SENSORY TRANSDUCTION
}

\section{A great TRP for hair cells}

The long search for the mechanosensitive transduction channel that allows hair cells in the mammalian inner ear to function might be over. Corey et al., writing in Nature, provide evidence in support of the idea that the channel, or at least a component of it, is the transient receptor potential (TRP) channel TRPA1.

Although it has been clear for some time that auditory transduction in vertebrates depends on the mechanical deflection of bundles of stereocilia on hair cells, and that this deflection opens ion channels in the tips of the stereocilia that are mechanically gated, the identification of these channels has proved tricky. However, the known permeability and conductance characteristics of the mechanosensitive channel are consistent with those of TRP channels, which are responsible for sensory transduction in other modalities including taste, thermal sensation and insect hearing.
Therefore, Corey and colleagues used in situ hybridization for all of the TRP channels in the mouse genome to search for one that was expressed in the mouse inner ear. TRPA1 was expressed in hair cells of both the cochlea and the vestibular system in mice, and its expression peaked at the developmental time point when these hair cells first become mechanosensitive. Antibody labelling showed that the cellular distribution of the channels was consistent with a role for TRPA1 in mechanotransduction in the stereocilia.

To test the idea that TRPA1 was involved in transduction, the authors used various methods to inhibit its expression. In zebrafish hair cells they used morphelino oligonucleotides, which inhibit the translation or splicing of mRNA, and in mouse hair cells they used adenoviruses to introduce small inhibitory RNAs (siRNAs) that targeted the TRPA1 message. In both cases, channel function was reduced, as measured either by dye accumulation or electrical responses (decreased microphonic potentials in zebrafish and decreased transduction currents in mouse cells).

In zebrafish, another TRP channel, Trpn1, is also required for hair-cell transduction, but this channel is not found in mammalian genomes. Corey et al. suggest that the two channels might form heteromeric channels in zebrafish. Interestingly, although they are not closely related phyogenetically, both TRPA1 and Trpn1 contain many ankyrin domains close to their amino termini. The authors propose that these repeats form a spring that could correspond to the 'gating spring' that has been biophysically identified as functioning in the hair-cell transduction channel complex. Yet another function for this versatile protein could come from its ability to undergo fast adaptation after opening. The rapid

\section{Steps to lasting plasticity}

A new study, reported in Science, shows how two proteins that are known to be important for long-term plasticity are linked. According to the results, tissue plasminogen activator
(tPA) activates plasminogen to form plasmin, which cleaves the precursor of brain-derived neurotrophic factor (BDNF) to release the mature neurotrophin.

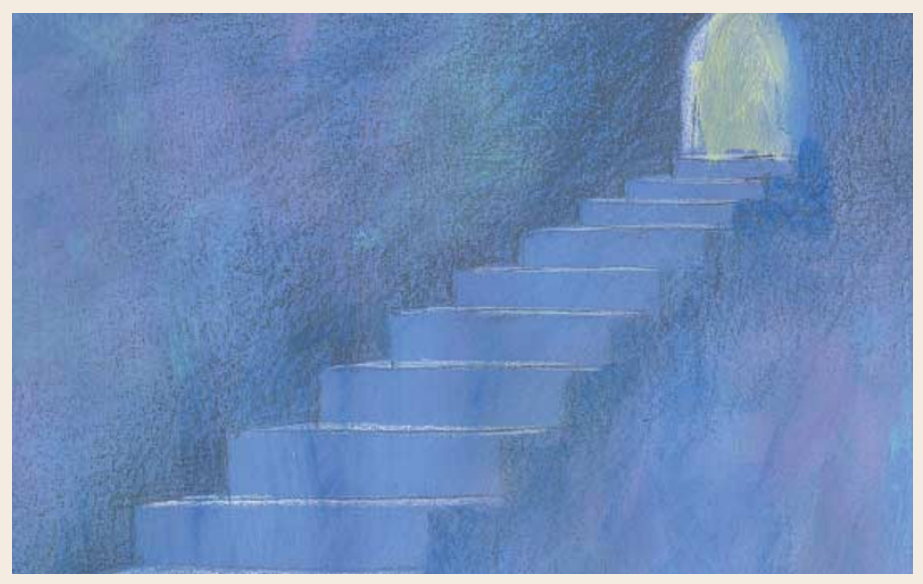

This process seems to be crucial for the late, protein-synthesisdependent phase of long-term potentiation (L-LTP).

Both BDNF and tPA have previously been found to be necessary for L-LTP in the hippocampus, but until now it has been unclear how they are related. There is in vitro evidence that plasmin, which is produced by tPA, can generate the mature form of BDNF by cleaving its precursor, proBDNF. So Pang et al. proposed that these two cleavage events, culminating in the production of BDNF, might be important stages in the expression of L-LTP.

Consistent with this idea, the authors found that the ability of protein synthesis inhibitors to 


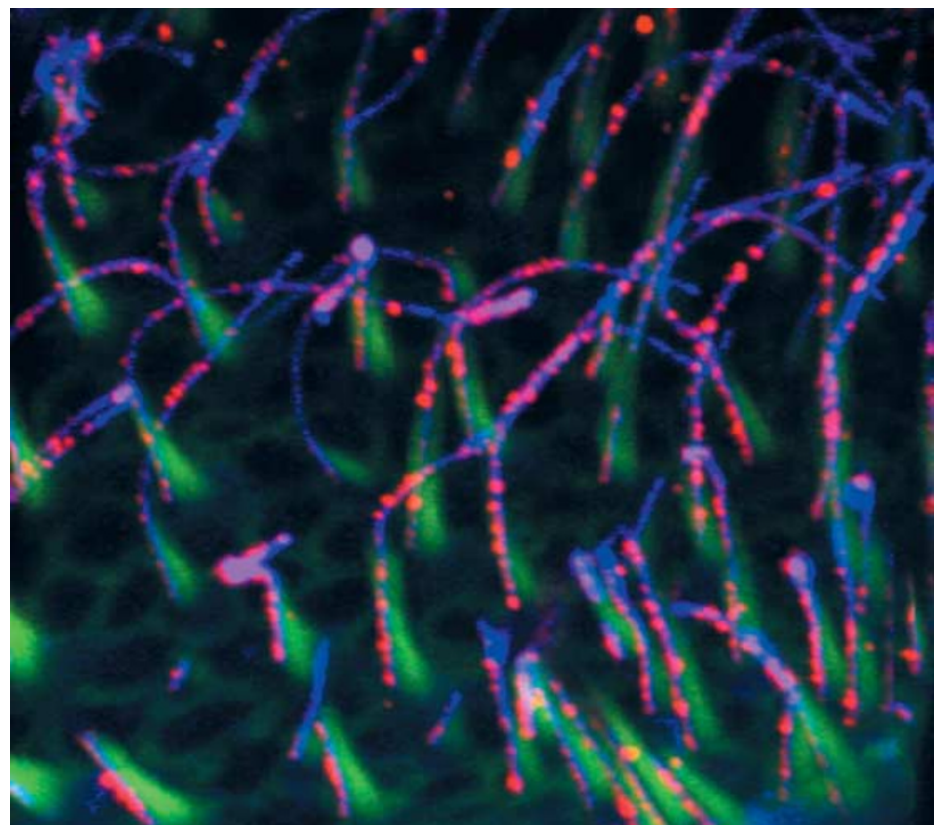

TRPA1 antibody labelling of hair cells in mouse semicircular canal. Red, TRPA1; blue, tubulin in kinocilia; green, actin in stereocilia. Image courtesy of D. Corey, Harvard Medical School, Boston, Massachusetts, USA.

conformational change that would result from channel closing might amplify the vibration of the basilar membrane in the cochlea. This 'cochlear amplifier' is believed to mediate frequency tuning. If TRPA1 turns out to represent the transduction channel, gating spring and cochlear amplifier in one, it would be a remarkable example of evolutionary ingenuity.

Rachel Jones

\section{(0) References and links} ORIGINAL RESEARCH PAPER Corey, D. P. et al. TRPA1 is a candidate for the mechanosensitive transduction channel of vertebrate hair cells. Nature 13 October 2004 (10.1038/nature03066) prevent hippocampal L-LTP could be blocked by an infusion of BDNF shortly after the LTP-inducing stimulus. BDNF treatment could also change early-phase (E-)LTP, induced by a different stimulation protocol, into L-LTP.

Mice lacking the gene for either tPA or plasminogen show impaired L-LTP. The authors found that this could be rescued by treatment with BDNF, consistent with the idea that the main role of tPA in L-LTP is to generate $\mathrm{BDNF}$.

Pang and colleagues also showed that, in vitro, plasmin cleaves proBDNF to form BDNF. Although neither plasminogen nor $\mathrm{PAA}$ alone could do this, when combined they were just as effective as plasmin, supporting the idea that IPA can indirectly promote proBDNF cleaveage by cleaving plasminogen to form plasmin. In addition, immunoprecipitation and immunochemistry studies showed that proBDNF is present at greater

\section{IN BRIEF}

\section{NEUROTECHNIQUE}

Second harmonic imaging of membrane potential of neurons with retinal.

Nemet, B. A. et al. J. Biomed. Opt. 9, 873-881 (2004)

The authors describe an optical method for imaging the membrane potential of neurons using all-trans retinal. When retinal is adsorbed to the neuronal plasma membrane, changes in membrane potential cause a change in its second harmonic generation that can be used to produce high-resolution images of live neurons. This study was carried out using cultured neurons, but it should be possible to extend the technique to acute brain slices.

\section{DEVELOPMENT}

Control of dendritic branching and tiling by the tricornered-kinase/furry signaling pathway in Drosophila sensory neurons.

Emoto, K. et al. Cell 119, 245-256 (2004)

Dendritic fields are often precisely controlled so that a given territory is completely covered by the dendrites of neighbouring neurons without any overlap. Emoto and colleagues show that the tiling of sensory neuron dendrites in Drosophila requires two evolutionarily conserved proteins - the tricornered (TRC) kinase and Furry (FRY). Flies in which either is mutated have dendrites with excessive branching and overlap. The evidence indicates that TRC and FRY promote repulsion between like dendrites and also limit dendritic branching.

\section{EYE MOVEMENTS}

concentrations in the hippocampi, and specifically the CA1 region, of mice that lack tPA.

By testing which of tPA, plasminogen and BDNF could rescue the L-LTP deficits in mice lacking each of these three proteins, the authors showed that plasmin is downstream of tPA in the induction of L-LTP, and BDNF is downstream of both. These results might indicate that BDNF is the crucial end-product of the proteinsynthesis-dependent step in L-LTP (and possibly long-term memory) induction. If that is the case, BDNF could be responsible for the structural and functional changes that are required for L-LTP.

Rachel Jones

(0) References and links ORIGINAL RESEARCH PAPER Pang, P. T. et al. Cleavage of pro-BDNF by tPA/plasmin is essential for long-term hippocampal plasticity. Science 306, 487-491 (2004) WEB SITE

Lu laboratory: http://neuroscience.nih.gov/ Lab.asp?Org_ID=275

\section{Dissociation of spatial attention and saccade preparation.}

Juan, C.-H. et al. Proc. Natl Acad. Sci. USA 101, 15541-15544 (2004)

It is unclear whether covert shifts in attention represent shifts in gaze that are planned but not executed, or an independent process. To address this question, Juan et al. measured deviations in saccades evoked by stimulation of the frontal eye fields in macaque monkeys during a pro-saccade or anti-saccade task. The results indicate that neurons in the frontal eye fields can covertly orient attention without preparing to carry out an eye movement.

\section{SYNAPTIC PLASTICITY}

\section{Single-shock LTD by local dendritic spikes in pyramidal} neurons of mouse visual cortex.

Holfhoff, K. et al. J. Physiol. 560.1, 27-36 (2004)

The dendrites of pyramidal neurons in layer 5 of mouse visual cortex show a variety of spiking activity, including spatially restricted spikes that are triggered by NMDA ( $N$-methyl-Daspartate) receptor activation. The authors use two-photon and confocal imaging to investigate these spikes, and find that they involve a high-amplitude, rapid calcium transient that is restricted to a small compartment of the dendrite. A single such spike, elicted by a single synaptic stimulus, can produce local, input-specific, long-term synaptic depression. 